\title{
Magnetic Properties and Electronic Structures of Compounds from the Hf-Co Phase System
}

\author{
Giorgio Concas $^{\mathrm{a}}$, Francesco Congiu ${ }^{\mathrm{a}}$, Jelena Belošević-Čavor ${ }^{\mathrm{b}}$, and Božidar Cekić ${ }^{\mathrm{b}}$ \\ ${ }^{a}$ Dipartimento di Fisica, Università di Cagliari and CNISM, S. P. Monserrato-Sestu km 0.700, \\ I-09042 Monserrato (Cagliari), Italy \\ ${ }^{\mathrm{b}}$ Institute of Nuclear Sciences Vinca, P. O. Box 522, 11001 Belgrade, Serbia \\ Reprint requests to Prof. G. C.; Fax: +39070510171; E-mail: giorgio.concas@dsf.unica.it
}

Z. Naturforsch. 62a, 452 - 456 (2007); received March 15, 2007

\begin{abstract}
Magnetic measurements of molar susceptibilities as a function of temperature in the temperature range between 5 and $400 \mathrm{~K}$ were performed for the intermetallic compounds $\mathrm{Hf}_{2} \mathrm{Co}$ and $\mathrm{HfCo}_{2}$, using a SQUID magnetometer. The density of states at the Fermi level for $\mathrm{Hf}_{2} \mathrm{Co}$ was evaluated from the measured spin paramagnetic susceptibility. In addition, band structure calculations using the augmented plane waves plus local orbitals (APW+lo) method as implemented in the WIEN2k programme package for the two compounds were done. The obtained results were compared with the measured data.
\end{abstract}

Key words: $\mathrm{Hf}_{2} \mathrm{Co}$; $\mathrm{HfCo}_{2}$; Augmented Plane Waves; Magnetic Susceptibility.

\section{Introduction}

Intermetallic compounds constitute a unique class of metallic materials, which are potential candidates of a new generation of materials due to specific magnetic, electrical, optical and structural properties at high temperatures in hostile environments [1-3]. Among the intermetallics of $3 \mathrm{~d}$ transition metals, those which exhibit a magnetic instability of the $3 \mathrm{~d}$ subsystem are of particular interest. The physical properties of these compounds provide a suitable test for various physical theories [4-7]. In this paper we focus our attention on two compounds from the Hf-Co phase system, $\mathrm{Hf}_{2} \mathrm{Co}$ and $\mathrm{HfCo}_{2}$, which have been investigated as interesting systems for applications as hydrogen storage, with high hydrogen to metal ratio, and for fabrication of cathodes for electrolytic hydrogen production $[8,9]$. Both compounds have cubic structure (space group $F d 3 m$ ); $\mathrm{Hf}_{2} \mathrm{Co}$ is of the $\mathrm{Ti}_{2} \mathrm{Ni}$-type, while $\mathrm{HfCO}_{2}$ is a Laves phase with the $\mathrm{C} 15\left(\mathrm{MgCu}_{2}\right.$-type $)$ structure [10].

The magnetic properties of $\mathrm{HfCO}_{2}$ have been investigated in the past $[4,11]$, and it is known since a long time that they are characterized by an exchange enhanced paramagnetism. The published results concerning the susceptibility $(\chi)$ showed differences in the temperature $(T)$ dependence at low temperature and a strong effect of deviations from the stoichiomet-

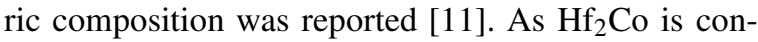
cerned, to the best of our knowledge there exists no investigation of the susceptibility vs. temperature in the literature.

Here we present molar susceptibility measurements as functions of the temperature for $\mathrm{Hf}_{2} \mathrm{Co}$ and $\mathrm{HfCo}_{2}$. In order to interpret the obtained results we have also performed ab-initio calculations of the electronic structure of the investigated compounds.

\section{Experimental}

Polycrystalline samples of $\mathrm{Hf}_{2} \mathrm{Co}$ and $\mathrm{HfCo}_{2}$ were obtained in a radio-frequency induction furnace under pure argon atmosphere, starting from high purity cobalt $(99.99 \%)$ and hafnium $(99.97 \%)$. Homogeneity was achieved by multiple remelting, in order to minimize the evaporation losses. By means of X-ray powder diffraction, the single-phase cubic structure was confirmed for $\mathrm{Hf}_{2} \mathrm{Co}$ and $\mathrm{HfCo}_{2}$. The cell parameter $a$ was experimentally determined as $12.066(1) \AA$ and 6.896(1) $\AA$ for the $\mathrm{Hf}_{2} \mathrm{Co}$ and $\mathrm{HfCo}_{2}$ samples, respectively.

The magnetization measurements were performed using a Quantum Design MPMS XL5 SQUID magnetometer equipped with a superconducting magnet producing fields up to $50 \mathrm{kOe}$ and calibrated using a Pd standard; the experimental data are given in units of 
Table 1. Structure parameters of $\mathrm{Hf}_{2} \mathrm{Co}$ and $\mathrm{HfCo}_{2}$. The distances are in $\AA$.

\begin{tabular}{|c|c|c|c|}
\hline & Number of bonds & Experimental (X-ray) & WIEN2k \\
\hline \multicolumn{4}{|l|}{$\overline{\mathrm{Hf}_{2} \mathrm{Co}}$} \\
\hline$u$ & & $0.2142(3)$ & 0.221 \\
\hline$v$ & & $0.816(1)$ & 0.813 \\
\hline \multicolumn{4}{|l|}{$16 \mathrm{c}$} \\
\hline Hf1-Co & 6 & $2.656(9)$ & 2.721 \\
\hline Hf1-Hf2 & 6 & $3.081(3)$ & 3.105 \\
\hline \multicolumn{4}{|l|}{$48 \mathrm{f}$} \\
\hline Hf2-Co & 2 & $2.77(2)$ & 2.645 \\
\hline $\mathrm{Hf} 2-\mathrm{Co}$ & 2 & $3.060(4)$ & 3.077 \\
\hline Hf2-Hf1 & 2 & $3.081(3)$ & 3.105 \\
\hline Hf2-Hf2 & 4 & $3.198(2)$ & 3.197 \\
\hline Hf2-Hf2 & 4 & $3.256(2)$ & 3.208 \\
\hline \multicolumn{4}{|l|}{$32 \mathrm{e}$} \\
\hline Co-Hf1 & 3 & $2.627(5)$ & 2.721 \\
\hline Co-Hf2 & 3 & $2.80(1)$ & 2.645 \\
\hline $\mathrm{Co}-\mathrm{Co}$ & 3 & $2.94(1)$ & 3.231 \\
\hline Co-Hf2 & 3 & $3.061(3)$ & 3.077 \\
\hline$a_{0}$ & & 12.0246 & 11.780 \\
\hline$B(\mathrm{GPa})$ & & & 204 \\
\hline \multicolumn{4}{|l|}{$\mathrm{HfCO}_{2}$} \\
\hline \multicolumn{4}{|l|}{ Hf } \\
\hline $\mathrm{Hf}-\mathrm{Co}$ & 12 & & 2.868 \\
\hline Hf-Hf & 4 & & 2.996 \\
\hline \multicolumn{4}{|l|}{$\mathrm{Co}$} \\
\hline $\mathrm{Co}-\mathrm{Hf}$ & 6 & & 2.868 \\
\hline $\mathrm{Co}-\mathrm{Co}$ & 6 & & 2.446 \\
\hline$a$ & & 6.896 & 6.710 \\
\hline$B(\mathrm{GPa})$ & & 220 & 238 \\
\hline
\end{tabular}

the Gauss CGS system. The magnetization was measured in an applied field of $50 \mathrm{kOe}$ in the temperature range 5-400 K; measurements at different fields were performed in order to correct the data for magnetically ordered impurities as described in [12].

\section{Band Structure Calculations}

The electronic structure calculations of $\mathrm{Hf}_{2} \mathrm{Co}$ and $\mathrm{HfCO}_{2}$ were performed using the augmented plane waves plus local orbitals (APW+lo) method as implemented in the WIEN2k programme package [13] within the local spin density approximation (LSDA) for the exchange-correlation potential [14]. Calculations were carried out with the plane wave cut-off parameter of 8.0 and 47 and $195 \mathrm{k}$ points in the irreducible wedge of the Brillouin zone for $\mathrm{Hf}_{2} \mathrm{Co}$ and $\mathrm{HfCO}_{2}$, respectively. The core-valence states separation was settled at $-7 \mathrm{Ry}$ in order to include low-lying Co 3 s states in the valence panel. The core states were treated fully relativistically, while the valence states were treated within the scalar relativistic approximation. The spin-orbit contribution was neglected. A volume optimization was done by performing a series of calculations, changing the volume within $\pm 10 \%$ of the experimental volume and calculating total energy as its function. For the $\mathrm{Hf}_{2} \mathrm{Co}$ structure, in addition to the volume optimization, it was necessary to relax the internal parameters, which was done by moving atoms along the symmetry directions until the HelmanFeynman forces were smaller than $5 \mathrm{mRy} / \mathrm{a}$. u. In our calculations self-consistency was achieved by demanding the convergence of the integrated charge difference between the last two iterations to be smaller than $10^{-5}$ electrons, since it ensures better stability of the calculated values than the corresponding energy criterion.

The calculated total energies were fitted to the Murnaghan equation of state [15] to obtain the equilibrium lattice constant and other structure properties. The interatomic distances were also calculated, using the equilibrium lattice constant and the space group of each compound. In Table 1, the equilibrium lattice constant $(a)$, bulk modulus $(B)$ and interatomic distances in the first coordination shell around each inequivalent atom in the structures are compared with the experiment $[16,17]$. The obtained lattice constants differ by about $3 \%$ from the experimental ones, while the calculated bulk modulus for $\mathrm{HfCo}_{2}$ is by about $8 \%$ larger than the measured one. The results concerning the bulk modulus for $\mathrm{Hf}_{2} \mathrm{Co}$, according to our knowledge, do not exist in the literature.

The calculated total densities of states (DOS) for $\mathrm{Hf}_{2} \mathrm{Co}$ and $\mathrm{HfCo}_{2}$ are given in Fig. 1, with the Fermi level marked with the dashed lines. It is seen that there is no band gap for both compounds, and the bonding nature is metallic since the DOS has a large finite value at the Fermi level. The estimated DOSs at the Fermi level are 14.66 and 7.48 states/eV per primitive cell, for $\mathrm{Hf}_{2} \mathrm{Co}$ and $\mathrm{HfCo}_{2}$, respectively, which corresponds to 1.83 and 1.87 states/(eV $\cdot \mathrm{Co}$-atom). As the electron density at the Fermi level $N\left(E_{\mathrm{F}}\right)$ can be related to the stability of a given compound, in such a way that the lower the $N\left(E_{\mathrm{F}}\right)$ the higher the stability $[18,19]$, this should indicate better stability of $\mathrm{Hf}_{2} \mathrm{Co}$ than of $\mathrm{HfCo}_{2}$. The $\mathrm{HfCo}_{2}$ Fermi level is placed well above the pseudo-gap, with some of the antibonding states being occupied, which also indicates its smaller stability.

\section{Experimental Results and Discussion}

Figure $2 \mathrm{a}$ shows the magnetic susceptibility of $\mathrm{Hf}_{2} \mathrm{Co}$ (lower curve) vs. temperature between 5 and $400 \mathrm{~K}$, determined from the magnetization at $50 \mathrm{kOe}$. The susceptibility shows only a weak de- 


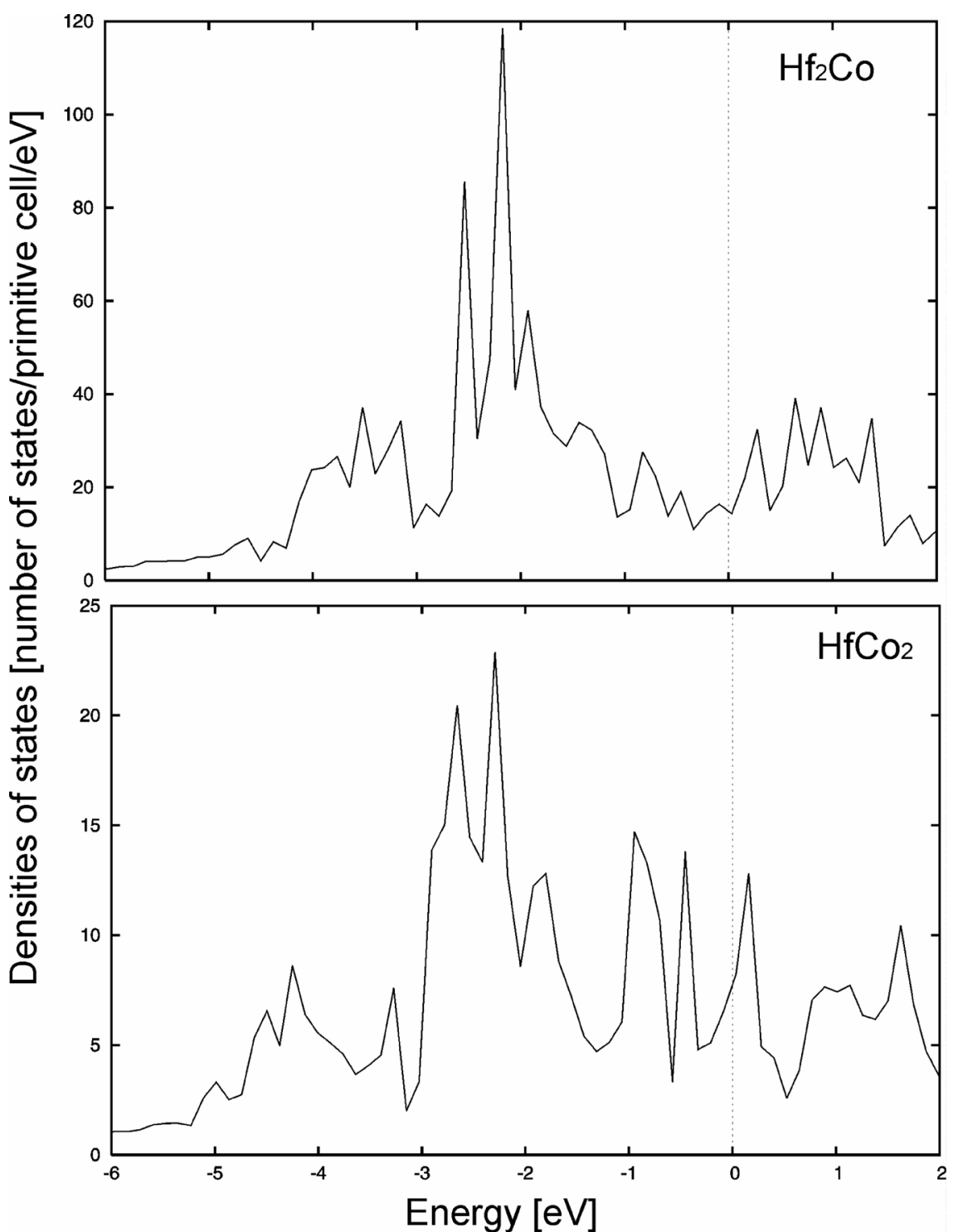

Fig. 1. Total densities of states of $\mathrm{Hf}_{2} \mathrm{Co}$ and $\mathrm{HfCO}_{2}$ in units of number of electrons per primitive cell per eV. The energy scale is in $\mathrm{eV}$, with the Fermi level at 0 . pendence on temperature; its value at $5 \mathrm{~K}$ is $(2.70 \pm$ $0.03) \cdot 10^{-4} \mathrm{~cm}^{3} / \mathrm{mol}$.

In the absence of paramagnetic Curie-like terms, the main contributions to the total magnetic susceptibility, $\chi_{\text {tot }}$, in a metal are given by $\chi_{\text {tot }}=\chi_{\text {core }}+\chi_{\mathrm{P}}+\chi_{\mathrm{L}}$, where $\chi_{\text {core }}$ is the diamagnetic core component and $\chi_{\mathrm{P}}$ and $\chi_{\mathrm{L}}$ are the Pauli and Landau-Peierls spin susceptibilities of the conduction electrons [20]. To separate the various terms appearing in the total susceptibility, we can first of all estimate the expected core diamagnetism, which can be evaluated using the calculated atomic diamagnetism of $\mathrm{Hf}^{4+}$ and $\mathrm{Co}^{2+}$ ions [21]. The core diamagnetic susceptibility has the value of -0.44 . $10^{-4} \mathrm{~cm}^{3} / \mathrm{mol}$ for $\mathrm{Hf}_{2} \mathrm{Co}$. Among the spin terms, in the case of $\mathrm{d}$ electrons treated in the tight binding approximation, the Pauli component $\chi_{\mathrm{P}}$ is of prime importance [22]. The Landau-Peierls diamagnetic susceptibility will be assumed equal to $-1 / 3 \chi_{\mathrm{P}}$, which is the expression in the case of free electrons [20].

The Pauli component $\chi_{\mathrm{P}}$ permits the evaluation of the density of states at the Fermi level $D\left(E_{\mathrm{F}}\right)$ by means of the relation

$$
\chi_{\mathrm{P}}=\mu_{\mathrm{B}}^{2} N D_{\chi}\left(E_{\mathrm{F}}\right),
$$




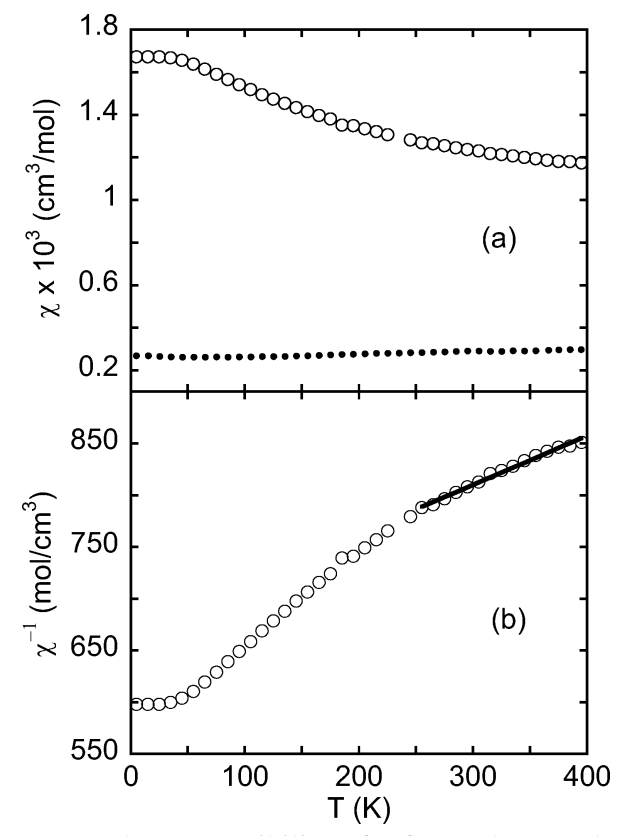

Fig. 2. (a) Molar susceptibility of $\mathrm{Hf}_{2} \mathrm{Co}$ (dots) and $\mathrm{HfCo}_{2}$ (open circles) between 5 and $400 \mathrm{~K}$ in CGS units. (b) Reciprocal molar susceptibility of $\mathrm{HfCo}_{2}$ (open circles) with the linear fit curve (solid line) for $T>250 \mathrm{~K}$.

where $\mu_{\mathrm{B}}$ is the Bohr magneton and $N$ is the Avogadro number; the apparent density of states $D_{\chi}\left(E_{\mathrm{F}}\right)$ appearing in the previous expression is enhanced with respect to the band value $D\left(E_{\mathrm{F}}\right)$ by the electron-electron interaction as described by the relation

$$
D\left(E_{\mathrm{F}}\right)=D_{\chi}\left(E_{\mathrm{F}}\right) /\left(1+I_{\mathrm{xc}} D_{\chi}\left(E_{\mathrm{F}}\right) / 2\right),
$$

in which $I_{\mathrm{xc}}$ is the effective exchange-interaction parameter [23, 24].

The evaluation of the density of states $D\left(E_{\mathrm{F}}\right)$ requires a calculated value of $I_{\mathrm{xc}}$. We used the value $I_{\mathrm{xc}}=0.99 \mathrm{eV}$ calculated for Co [25], because Co gives the main contribution to the DOS at the Fermi level. The resulting value is $D\left(E_{\mathrm{F}}\right)=1.77$ states $/(\mathrm{eV} \cdot \mathrm{Co}-$ atom); the comparison with the value obtained by band calculations in this work [1.83 states $/(\mathrm{eV} \cdot \mathrm{Co}$-atom) shows that the calculations give an acceptable agreement with the experiment.

The magnetic susceptibility of $\mathrm{HfCo}_{2}$ (upper curve) vs. temperature is shown in Figure 2a. It is strongly temperature-dependent and reaches its maximum value for $T \rightarrow 0 \mathrm{~K}$; above this temperature the susceptibility decreases. Its value at $5 \mathrm{~K}$ is $(1.67 \pm 0.02)$.
$10^{-3} \mathrm{~cm}^{3} / \mathrm{mol}$. Following the procedure described for $\mathrm{Hf}_{2} \mathrm{Co}$, the density of states was evaluated starting from the experimental susceptibility for $T \rightarrow 0 \mathrm{~K}$ $\left[(1.67 \pm 0.02) \cdot 10^{-3} \mathrm{~cm}^{3} / \mathrm{mol}\right]$; the obtained value is $D\left(E_{\mathrm{F}}\right)=1.92$ states $/(\mathrm{eV} \cdot \mathrm{Co}$-atom $)$. The value of 1.87 states/(eV $\cdot$ Co-atom) obtained by band calculations in this work agrees well with the experimental value.

The reciprocal susceptibility $\chi^{-1}$ vs. temperature is plotted in Figure $2 \mathrm{~b}$. The linear behaviour of $\chi^{-1}$ in the high temperature region $(T>250 \mathrm{~K})$ shows that the susceptibility follows a Curie-Weiss-like expression $\chi=A /(T-B)$; the linear fit of $\chi^{-1}$, plotted in Fig. $2 \mathrm{~b}$, gives the constants $A=(2.11 \pm$ $0.01) \mathrm{cm}^{3} \cdot \mathrm{K} / \mathrm{mol}$ and $B=(-1408 \pm 1) \mathrm{K}$.

The large value of the paramagnetic susceptibility of $\mathrm{HfCo}_{2}$ and its strong temperature dependence are associated with a spin susceptibility enhanced by the effect of spin fluctuations $[26,27]$. The evolution of $\chi$ vs. temperature in $\mathrm{ACo}_{2}$ compounds (where $\mathrm{A}$ is a transition metal element of the group IIIB or IVB) has been theoretically investigated by Yamada et al. [27]; it has been interpreted starting from the shape of the density of states of d electrons near the Fermi energy. If $E_{\mathrm{F}}$ is above a sharp bonding peak of the DOS, $\chi(T)$ shows a maximum at finite temperature; if $E_{\mathrm{F}}$ is near a smaller antibonding peak, $\chi(T)$ reaches its maximum value for $T \rightarrow 0 \mathrm{~K}$ and follows a Curie-Weiss-like expression in the high temperature range [27]. The DOS of $\mathrm{HfCo}_{2}$ presented in Section 3 corresponds to the second case, which agrees with the experiment because $\chi(T)$ measured in this work shows the foreseen temperature dependence.

\section{Conclusions}

In this paper we have studied $\mathrm{Hf}_{2} \mathrm{Co}$ and $\mathrm{HfCo}_{2}$, both experimentally and theoretically, using ab-initio calculations. The molar susceptibilities as a function of temperature were measured using a SQUID magnetometer. We have also reported densities of states and structure parameters for the mentioned compounds. The calculated results are in fair agreement with the experimental ones.

\section{Acknowledgements}

This work has been partly supported by the Serbian Ministry of Science and Environmental Protection under the project $141022 \mathrm{G}$. 
[1] M. Nakamura, in: Intermetallic Compounds (Eds. J. H. Westbrook, R. L. Fleischer), Wiley and Son Co., New York 2000, p. 1.

[2] G. Sauthoff, in: Intermetallic Compounds (Eds. J.H. Westbrook, R. L. Fleischer), Wiley and Son Co., New York 2000, p. 41.

[3] G. Sauthoff, in: Oxidation of Intermetallics (Eds. H. J. Grabke, M. Schutze), Wiley-VCH, New York 1997, p. 3.

[4] E. Burzo, E. Gratz, and V. Pop, J. Magn. Magn. Mater. 123, 159 (1993).

[5] E. Gratz and A. S. Markosyan, J. Phys. Condens. Mat. 13, R385 (2001).

[6] T. Moriya, J. Magn. Magn. Mater. 14, 1 (1979).

[7] E. Burzo and R. Lemaire, Solid State Commun. 84, 1145 (1992).

[8] D. Mukai, H. Miyata, and K. Aoki, J. Alloys Compounds 293-295, 417 (1999).

[9] D. Stojić, Š. Miljanić, B. Cekić, V. Koteski, J. Belošević-Čavor, and M. Marčeta Kaninski, International Hydrogen Energy Congress (IHEC'2005), July $13-15,2005$, Istanbul, Turkey (Proceedings on CD).

[10] R.W.G. Wyckoff, Crystal Structures, Interscience Publishers, New York 1966.

[11] Y. Aoki, T. Nakamichi, and M. Yamamoto, Phys. Status Solidi B 56, K17 (1973).

[12] J. Belošević-Čavor, F. Congiu, V. Koteski, B. Cekić, and G. Concas, Mater. Sci. Forum 518, 319 (2006).

[13] P. Blaha, K. Schwarz, G. K. H. Madsen, D. Kvasnicka, and J. Luitz, WIEN2k, An Augmented Plane Wave Plus Local Orbitals Program for Calculating Crystal
Properties, Vienna University of Technology, Vienna, Austria 2001.

[14] P. Hohenberg and W. Kohn, Phys. Rev. Lett. 77, 3865 (1996).

[15] F. D. Murnaghan, Proc. Natl. Acad. Sci. USA 30, 244 (1944).

[16] B. Cekić, D. Rodić, N. Ivanović, M. Mitrić, S. Koički, and M. Manasijević, Matica Srpska, Proc. Natural Sci. 85, 243 (1993).

[17] K. C. Chen, F. Chu, P. G. Kotula, and D. Thoma, Intermetallics 9, 785 (2001).

[18] J. H. Xu and A. J. Freeman, Phys. Rev. B 41, 12553 (1990).

[19] R. Kuentzler and R. M. Waterstrat, Solid State Commun. 68, 85 (1988).

[20] A. H. Morrish, Physical Principles of Magnetism, Wiley, New York 2001.

[21] R. R. Gupta, in: Landolt-Bernstein, Vol. II/16 (Eds. K. H. Hellwege and A. M. Hellwege), Springer, Berlin 1986, p. 402.

[22] A. M. Klogston, W. Jaccarino, and Y. Yafet, Phys. Rev. 134, A650 (1964).

[23] E. C. Stoner, Proc. R. Soc. London A 154, 656 (1936).

[24] O. K. Andersen, W. Klose, and H. Nohl, Phys. Rev. B 17, 1209 (1978).

[25] O. Gunnarsson, J. Phys. F 6, 587 (1976).

[26] H. Yamada, J. Inoue, K. Terao, S. Kanda, and M. Shimizu, J. Phys. F 14, 1943 (1984).

[27] H. Yamada, J. Inoue, and M. Shimizu, J. Phys. F 15, 169 (1985). 